\title{
Anamorphic Experiences in 3D Space: Shadows, Projections and Other Optical Illusions
}

\author{
Ioanna Symeonidou ${ }^{1,2}$
}

\begin{abstract}
The paper presents recent research on the reconstruction of Anamorphic effects and other optical illusions, shadows and projections, with the use of CAD systems. The first part of the paper is a bibliographical overview about the appearance of optical illusions in art, ranging from the work of Niceron to the extravagant sculptures of contemporary artists such as Markus Raetz. The second part of the paper reports on an educational approach that introduces anamorphic geometries into the teaching of digital methods of representation at Graz University of Technology. There is an overview of the experiments and methodology for constructing optical illusions in a CAD environment as well as examples drawn from student projects. The paper concludes with some observations and remarks relating to the aforementioned educational experience.
\end{abstract}

Keywords Projections · Optical illusions · CAD · Didactics · Art · Anamorphosis · Digital representations

\section{Introduction}

The understanding of space through diverse projections and the study of shadow has ordinarily formed part of the curriculum of architectural education. Courses of descriptive geometry traditionally taught with compass and straightedge, are currently enriched with digital media, introducing the ever-growing field of computational geometry. Based on this propaedia we are now moving a step further, utilizing a new vocabulary of anamorphic effects, optical illusions and anagrams

Ioanna Symeonidou

i.symeonidou@ihu.edu.gr

1 Graz University of Technology, Graz, Austria

2 International Hellenic University, Thessaloniki, Greece 
which can result in fascinating spatial experiences. In this a game of perception a spectator in either physical or digital space might experience confusion or surprise walking through spaces that look different than what they really are! Nevertheless, the so-called "illusion" always comes down to simple geometric rules. All the aforementioned effects have a concrete geometric explanation. This paper presents research into examples of optical illusion and their digital reconstruction, and an educational process that attempts to understand, analyze, and construct optical illusions and visually ambiguous spaces.

\section{Background and Motivation}

Within the agenda of the course "Digital Methods of Representation" at Graz University of Technology, students were asked to employ their skills in descriptive geometry in new ways. MySpace was a course about digital representation held at the Institute of Architecture and Media during summer semester 2013. The course introduced architecture students to 3D modeling in Rhinoceros software. Students had some previous experience with CAD software and also with descriptive geometry, and in the aforementioned course they were asked to combine their previous knowledge and use 3D modeling in new ways. The aim of the course was not to offer a mere software tutorial but to challenge the students to use $3 \mathrm{D}$ modeling in new creative ways, challenging the notion of perception, understanding the space and reconstructing spatial uncertainties. The thematic area of threedimensional optical illusions offered a very fertile ground for such explorations and generated a great variety of student projects while teaching about projections, shadows, Boolean operations and advanced surface editing. This paper reports on the methods developed during this course and the obtained results.

The semester-long course was structured in units of one or 2 weeks duration, conducting a specific sequence of exercises and tutorials, with intermediate submissions during the entire semester. The course was based on a long tradition of similar courses held at the Institute of Architecture and Media, always though exploring a different thematic area. This year the course MySpace was focusing on developing design strategies that challenge the viewer's understanding of space, comprehending the links between geometry and perception.

\section{A Brief Introduction to Optical Illusions}

An optical illusion or visual illusion is characterized by visually perceived images that differ from objective reality. The most common ones are certain 2D configurations that trick the eye, giving a false impression about lengths, inclinations of lines, or tonality of colours. In 3D space, optical illusions usually refer to our ability to perceive perspective views, the understanding of light and shadow as well as our wrongly perceived distance from certain objects. More specifically, Anamorphosis is a distorted projection of an image or object, which only becomes clear when the observer's point of view originates from the so-called 
vantage point or it is viewed as a reflection produced by special devices, such as a specific curved mirror surface. The word "anamorphosis" originates from the Greek compound word, whose prefix ana-means back or again, and the word-morphe, which means shape or form. There are two main types of anamorphosis: perspective or oblique, and mirror or catoptric from Greek catoptron, which means mirror.

The earliest example of perspective anamorphosis is the famous Leonardo's Eye by Leonardo Da Vinci in 1485. These techniques were further advanced by Renaissance artists who gradually mastered the skill of distorting and stretching images and started introducing anamorphic images in their paintings. One of the most prominent examples is Holbein's painting The Ambassadors (1533) with a Memento mori anamorph skull in the foreground (Fig. 1). The catoptric anamorphic images appeared a bit later during the sixteenth century. These employed certain optical machines, usually a cylindrical or conical mirror, and were initially established as a way to picture hidden meanings in painting.

Anamorphic drawings become particularly famous in the mid-seventeenth century with the work of French mathematician and artist Jean-François Niceron (Niceron 1638; Hunt 2006) and his book on La Perspective Curieuse. As Niceron

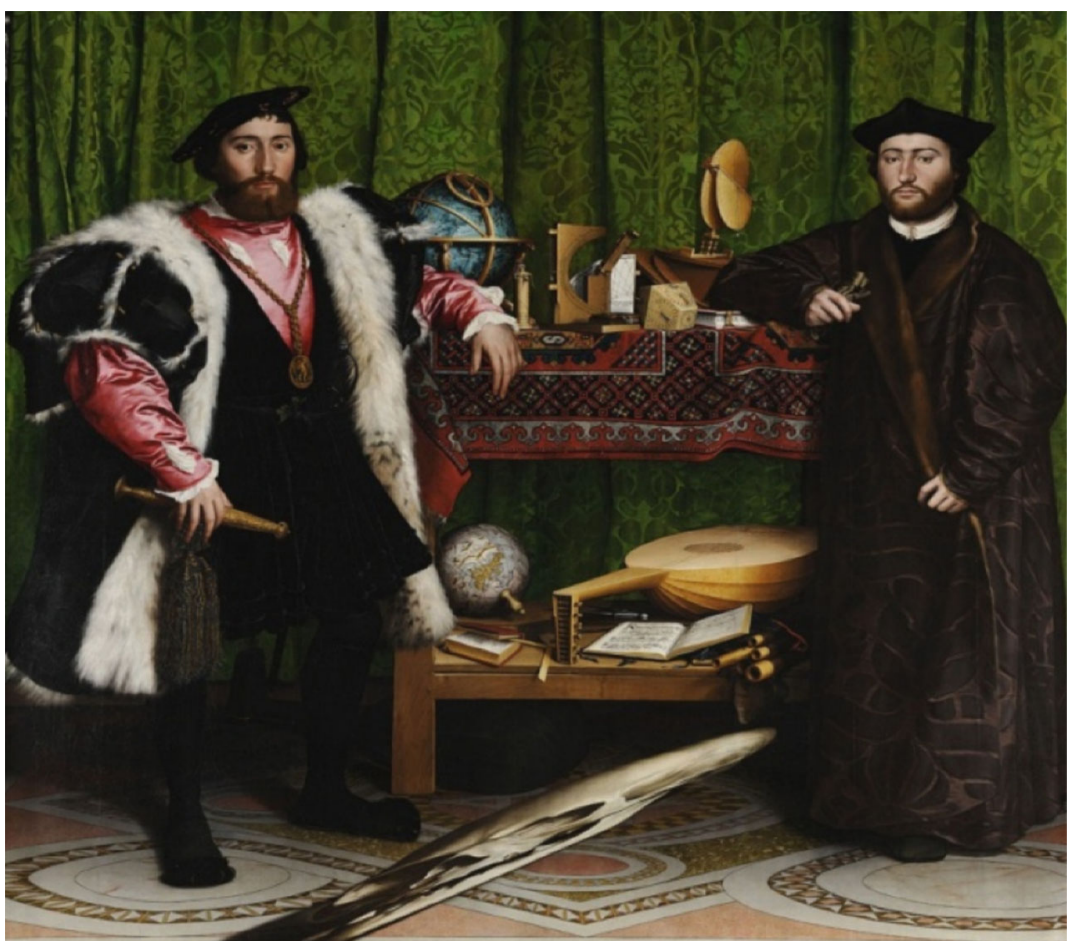

Fig. 1 The Ambassadors (1533) by Hans Holbein the Younger-Image source https://commons. wikimedia.org/wiki/File:Hans_Holbein_the_Younger_The_Ambassadors_Google_Art_Project.jpg. This is a faithful photographic reproduction of a two-dimensional, public domain work of art 

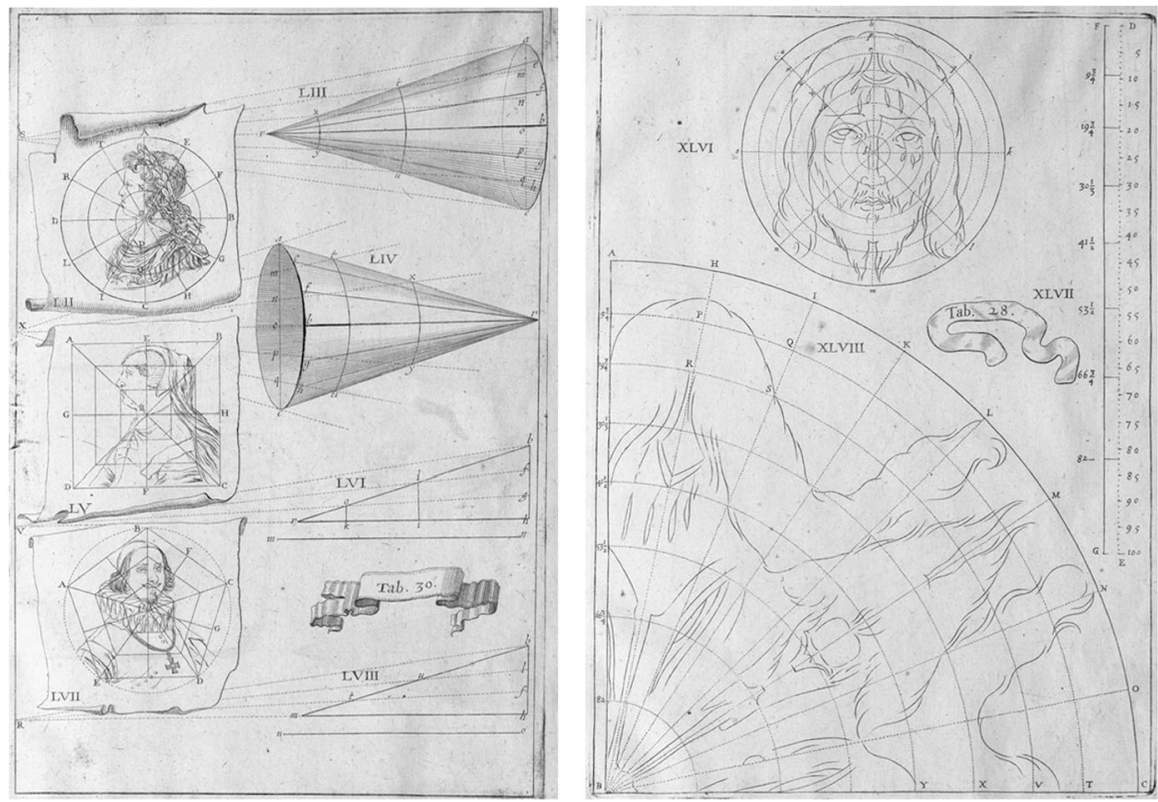

Fig. 2 Notes on conical anamorphosis by Jean-François Niceron from the book on La Perspective Curieuse-Image sources https://www.flickr.com/photos/bibliodyssey/7806885986 and https://www. flickr.com/photos/bibliodyssey/7806885654 Licenced under Creative Commons Licence-Attribution: https://creativecommons.org/licenses/by/2.0/. The pictures have not been modified

explains, the original figure is set in a grid and it is translated square by square to the deformed grid (Fig. 2).

\section{Artistic References and Objectives}

One of the objectives of this research was to study certain three-dimensional optical illusions in order to understand the relationship between geometry and perception. The students that took part in this course used the underlying principles of the examples presented to work on their own case studies as part of the course requirements. Among the aims of MySpace was to create experiences/environments of illusion and surprise through the design of geometrically complex objects and spaces with the use of digital media. Students were challenged to create ambiguous objects where 3D geometry would manifest different meanings when perceived from different points of view. Using 2D curves from numbers or letters as initial input, they would create and edit solids aiming at the design of an Ambigram (Hofstadter 1987). An Ambigram is a word, symbol or artistic form, consisting of one or more $3 \mathrm{D}$ elements; it can be understood or read in more than one way when viewed from a different direction, perspective or orientation. This first exercise introduced students to the geometry of projections, the perspective and orthographic views and the editing of solids. 
Ambigrams can fall into several categories, ranging from natural, to rotational, to 3-dimensional. One of the most famous ambigrams is the one created by Douglas Hofstadter, American professor of cognitive science, who also coined the term (Polster 2000). For the cover of his book Gödel, Escher, Bach: an Eternal Golden Braid (Hofstadter 2000), where each of the 3 projections to the Cartesian planes recreate the silhouette of the three initial letters, "G", "E" and "B" representing visually the connections and reciprocities among the works of Gödel, Escher and Bach. Following the same lines and going into mathematics and computation, the sqriancle (SQuare, tRIANgle and cirCLE, according to Sela and Elber 2007) is an object that resembles a square, a triangle and a circle when viewed from three different angles. Understanding the underlying geometrical principles led to the construction of ambigrams for the first set of projects to be undertaken during the MySpace course at Graz University of Technology. The methodology followed will be explained in detail in the following section of the paper.

Other explorations within the agenda of the aforementioned course involved the understanding of optical illusions, shadows and anamorphic drawings in line with the artwork of conceptual artists such as the shadow sculptures and optical illusions of Shigeo Fukuda and Kumi Yamashita, the ambigrams of Markus Raetz (Fig. 3) and the anamorphic typography of Thomas Quinn (Fig. 4) among others. In all of

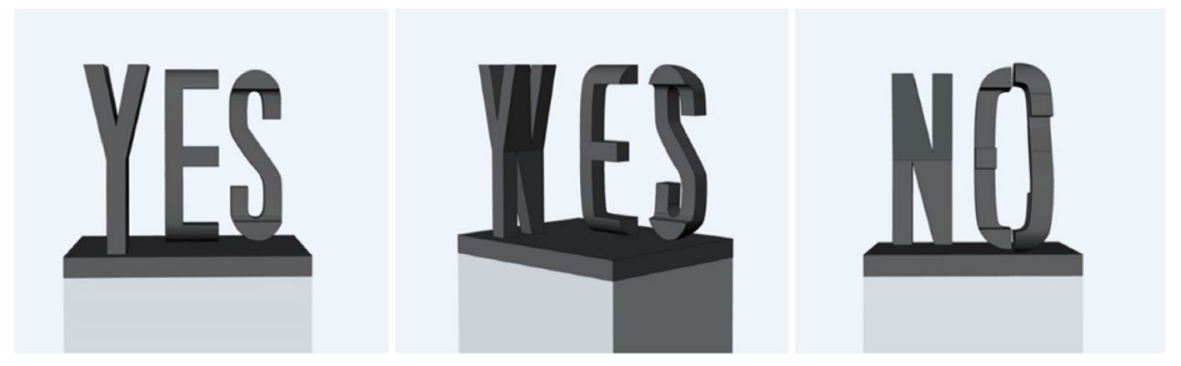

Fig. 3 Digital 3D reconstruction of the Ambigram Sculpture by Markus Raetz modeled by the author. The sculpture was modeled in Rhino 3D. The modeling process is shown in Fig. 5

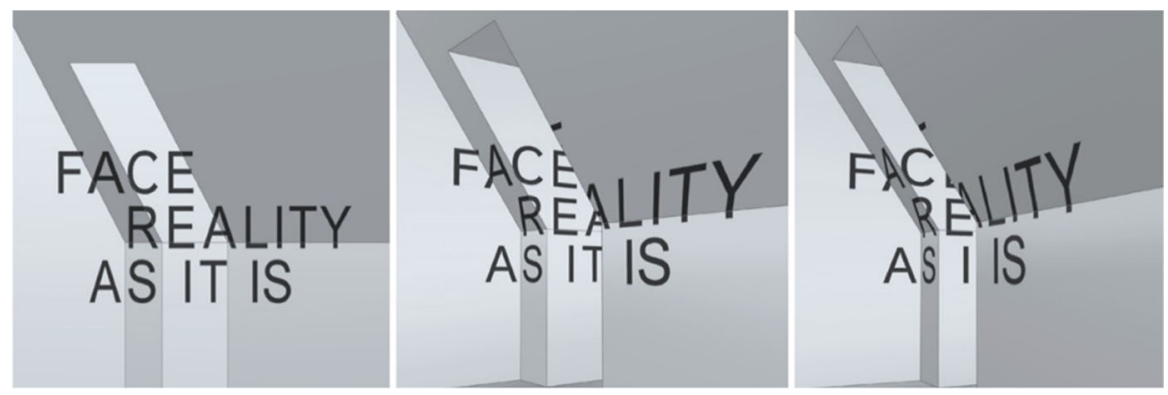

Fig. 4 Digital 3D reconstruction of the Anamorphic Typography installation by Thomas Quinn modeled by the author. The sculpture was modeled in Rhino 3D. The modeling process is shown in Fig. 6 


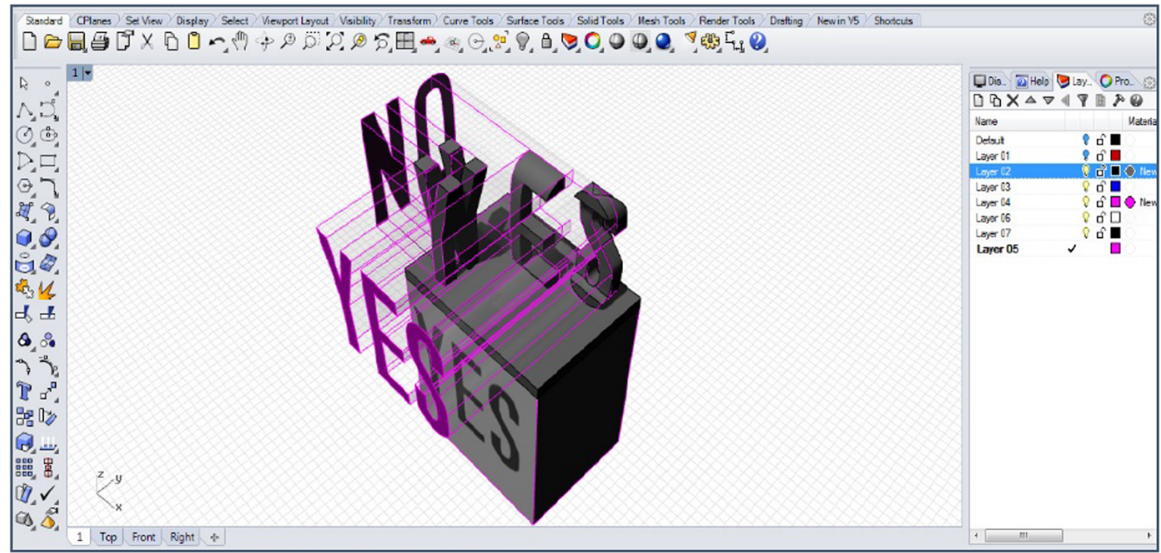

Fig. 5 Modeling process of the Ambigram by Markus Raetz in Rhinoceros 3D. The words YES and NO are modeled as 2D curves on two perpendicular planes. The 2D curves are extruded straight (seen in translucent) and the intersection of the solids results in the final geometry of the sculpture

the above cases the key concept is the projection of the geometry on different planes.

Markus Raetz is following the lines of Douglas Hofstadter's ambigrams. The artist goes one step further, by sculpting words of opposing meanings onto a unique piece. As with the ambigrams, the words can be read when the viewer is situated on the appropriate position with regards to the sculpture and the process can be better perceived when moving around the artifact. Again the key concept relates to the projections of the $3 \mathrm{D}$ object upon an imaginary plane that is perpendicular to the viewer's eye. This can be also seen in the $3 \mathrm{D}$ modeling process, where the solid intersection of the two projections results in the sculptural form (Fig. 5). The work of Markus Raetz however, is not limited to the sculptures of words of opposing meanings. The rabbit and the man is another example where topologically equal, yet geometrically different entities are combined in one sculpture which has been exhibited either as a surface or as a wireframe, often presented together with a mirror placed at an appropriate distance and angle to the sculpture so as to make the whole concept visible at a glance.

Likewise, in the work of Thomas Quinn, although it is based on the same principles of projection parallel to the eye of the spectator, the projected shapes fall on different planes that meet in certain angles. This creates a more fragmented visual result. When standing very near the artwork it is not easy to perceive the intended shape. The work of Quinn is often referred to as Anamorphic Typography, as the meaning of the dissected words can be only perceived from a certain distance and a certain point in space, the so-called vantage point (Fig. 4). The projection of the words on the tilted walls is seen in Fig. 6.

Similar to Quinn, the work of Felice Varini, which was presented and analyzed by a recent Nexus Network article (Di Paola et al. 2015), also explores the issue of transformation and is closely linked to an architectural framework. It is the spectator 


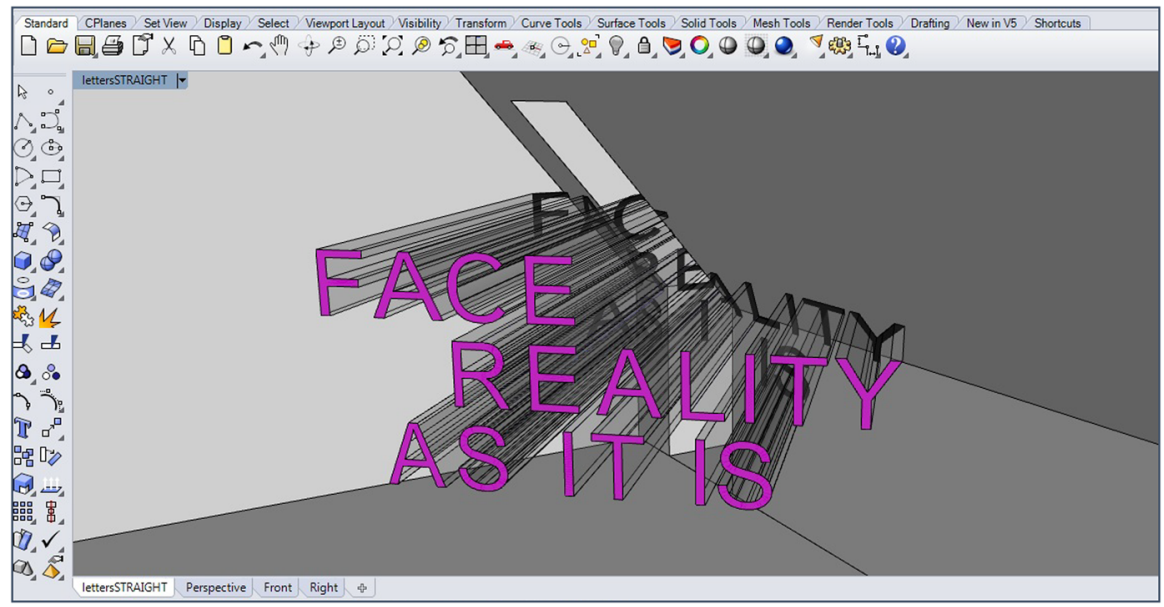

Fig. 6 Modeling process of the Thomas Quinn's Anamorphic Typography in Rhinoceros 3D. The letters are constructed on a new Construction Plane (CP) which is $45^{\circ}$ rotated with regards to the original wall. The letters are then projected to the wall, and as a result parts of the projection fall on different planes, creating the optical illusion

who moves within the architecture to find the point of "encounter with the work", where the components are assembled to represent a precise figure. Varini's work is related to the surrounding architecture, as he paints on buildings, urban spaces, vertical and horizontal surfaces. The characteristic of his paintings is the unique Vantage Point from which the viewer can see the complete work of art, which usually consists of simple geometric shapes. If not standing at the vantage point, the viewer will only perceive fragmented shapes. The construction process of the works of Quinn and Varini follows the same principles of projection onto irregular surfaces, i.e. on surfaces that are either connected or not, located in variable distances from the Vantage Point.

Just as the work of Quinn and Varini is an interpretation and extrapolation of perspective or oblique anamorphosis in 3D space, so is the work of British artist Jonty Hurwitz translating the mirror or catoptric anamorphosis in actual sculptures. The artist utilizes 3D scans of bodies or animals, which are subsequently distorted according to $\mathrm{Pi}$. The reflection of the sculptures on a cylindrical mirror recreates the model as it would look if none of the transformations had taken place. Cylindrical Mirror Anamorphosis has been studied in depth by Čučaković and Paunović, and their work is documented in their article included in the 17th volume of the Nexus Network Journal (Čučaković and Paunović 2015). They study urban installations and describe their workflow, which ranges from experiments to digital methods undertaken in AutoCAD 3D.

In 2013, an international exhibition in Dublin, featuring also work by Jonty Hurwitz (Figs. 7, 8), brought together several contemporary artists that deal with the theme of optical illusions. The exhibition titled ILLUSION: Nothing is as it seems took place at Science Gallery, Trinity College Dublin from July to September 2013. 

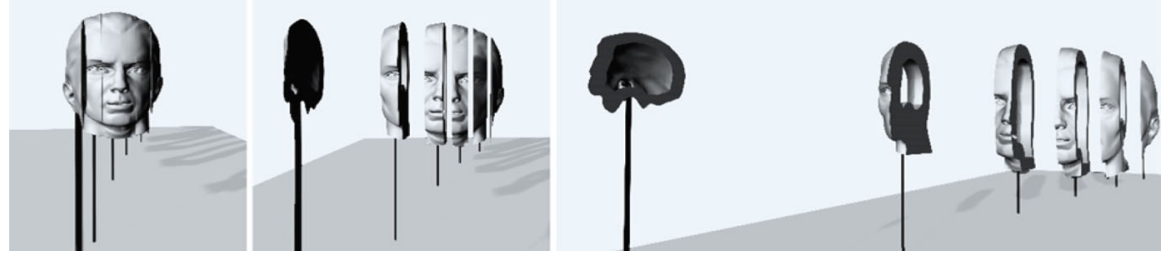

Fig. 7 Digital 3D reconstruction of the Hurwitz Singularity, an anamorphic sculpture by Jonty Hurwitz modeled by the author

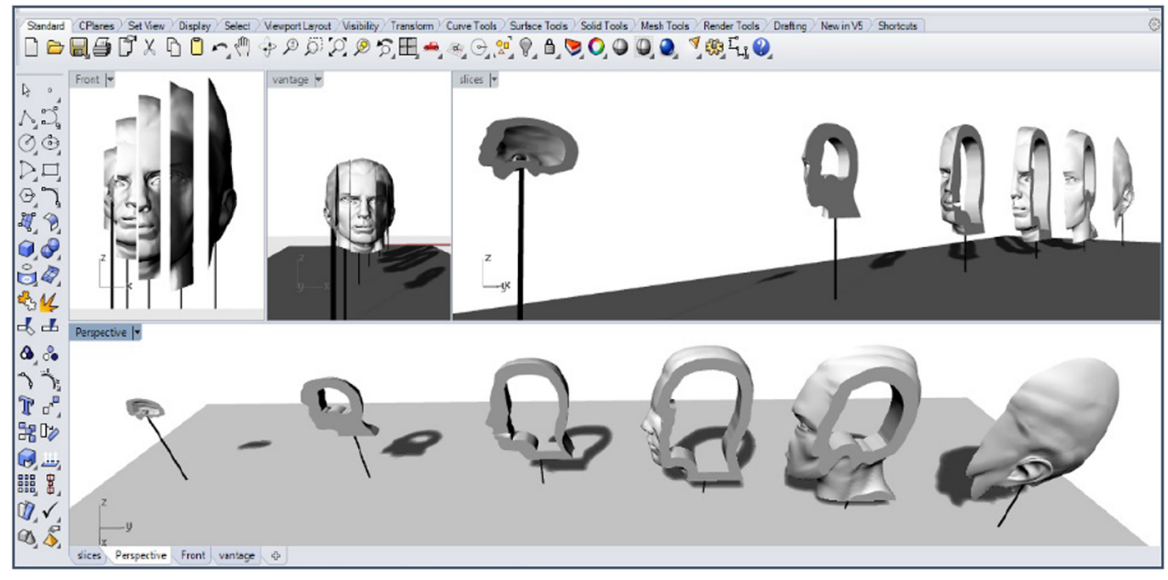

Fig. 8 Modeling process of the Hurwitz Singularity in Rhinoceros 3D. On the viewports we can observe the differences between the front view (a) and the perspective view through the Vantage Point (b), as well as side view (c) and tilted top view (d), where one can observe the reduction of size of the slices, so that the face is correctly seen from the Vantage Point

According to the curators, "Illusions distort the senses and mystify our logical thinking. The human mind can be easily fooled. This exhibition joins magic with psychology, optical illusions with scientific reasoning and confusion with clarity" (Illusion: Nothing is as it seems 2013). The design and construction of an optical illusion often requires a multidisciplinary approach consisting of psychologists, biologists, mathematicians, geometers and other scientists.

Another important aspect of optical illusions, and which also resulted in a series of exercises during this course, was the understanding of the geometry of shadow and the implementation of shadows in an architectural context to accentuate the volumetric configuration of a building. The study of shadows has traditionally formed part of the architectural curriculum, though before the introduction of digital media, the exercises undertaken by compass and straightedge were usually limited to simple volumes and common architectural elements such as columns, beams, inclined roofs, etc (Figs. 9, 10). It would require an extremely high level in 


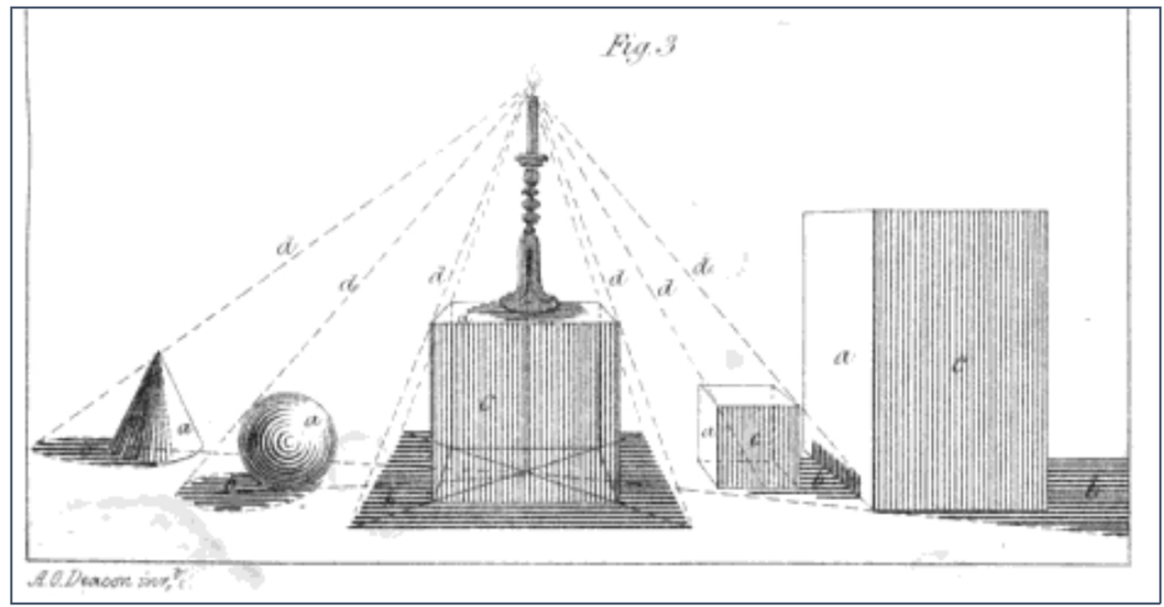

Fig. 9 Construct a shade from a single light source, from the book Elements of perspective drawing, MDCCCXLI (1841) by Augustus Deacon_Image source https://commons.wikimedia.org/wiki/File: Construct_a_shadow_from_a_single_light_source.png Licenced under Creative Commons LicenceAttribution: https://creativecommons.org/licenses/by/2.0/. The picture has not been modified

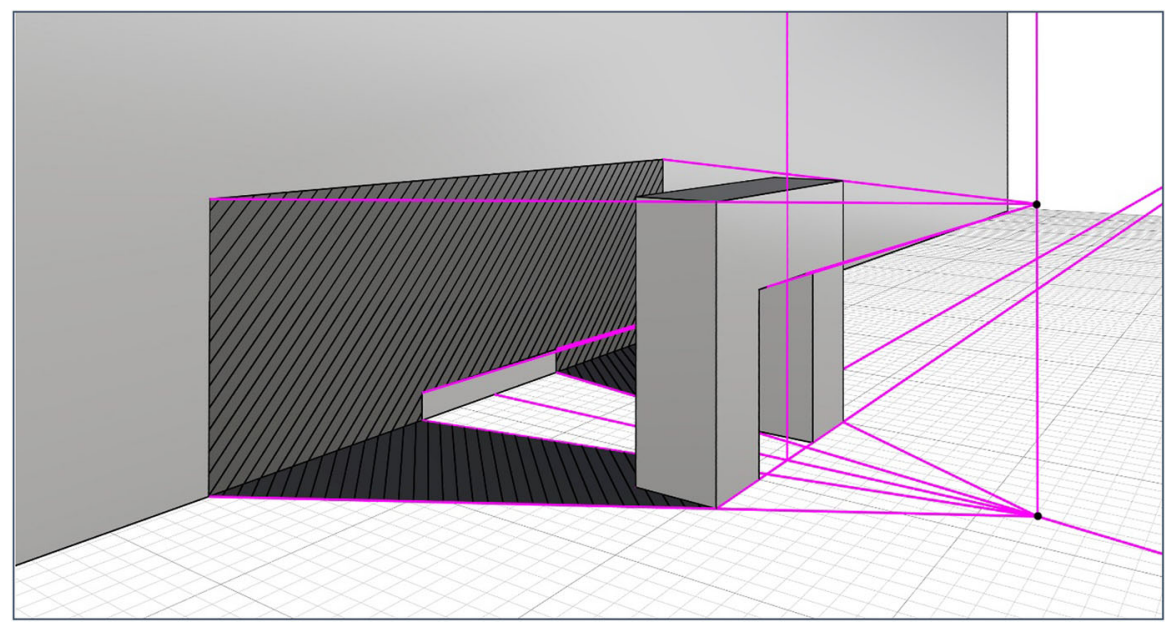

Fig. 10 Computerized traditional modeling process for shadow construction, where the shadow falls on two perpendicular planes

descriptive geometry to calculate the shadow of a complex free-form structure onto an equally nonstandard background. However with the use of digital media, such calculations and geometric constructions can be done quickly and accurately. As the aim was to learn about the geometric construction of shadows and implement them in their projects, a new design motivation emerged. The students would calculate the shadows that a certain object casts on a non-standard background, and will utilize the shadow as a primitive for developing their own design from it. 
An interesting reference for this series of explorations has been the project Shadow Construction by Marte Haverkamp, where the artist reconstructs absurd geometrical artifacts, deriving from the shadows that everyday objects drop on irregular surfaces. As the artist explains, "the shadows follow the floors, corners [...] They appear to be fluid, so smooth they run on different surfaces. She lets the shadow of existing products fall on different surfaces and thus she creates new objects in which "the intangible becomes tangible" (Haverkamp 2012). In a similar approach, within the digital environment, the aim was to construct shadows of a much bigger complexity and with a greater degree of detail. Such a task, as discussed earlier, would have been very difficult (if not impossible) to bring forward and accurately calculate with analogue media. The research was initiated with the intention of constructing a shadow that only when seen from the Vantage Point may recall the original object. When seen from any other perspective it is difficult to recognize the shape of the initial object. For this experiment, we utilized iconic design objects that are easily recognizable by their silhouette, such as the Carrera glasses (Fig. 11) and the Barcelona chair (Fig. 12).
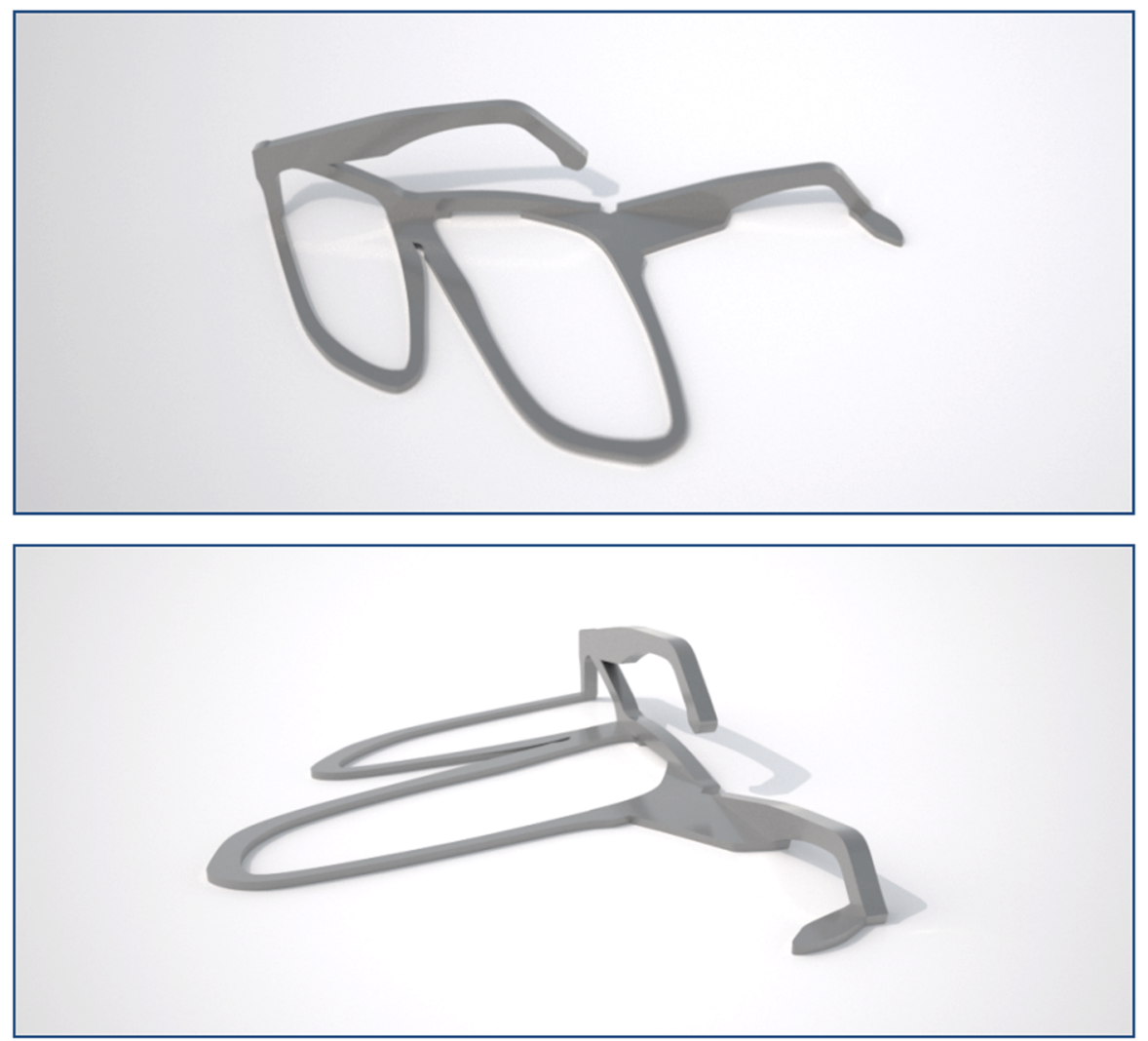

Fig. 11 Digital 3D model of an anamorphic shadow of a pair of Carrera glasses modeled by the author. Image (a) seen from the vantage point, image (b) is seen from a different perspective 

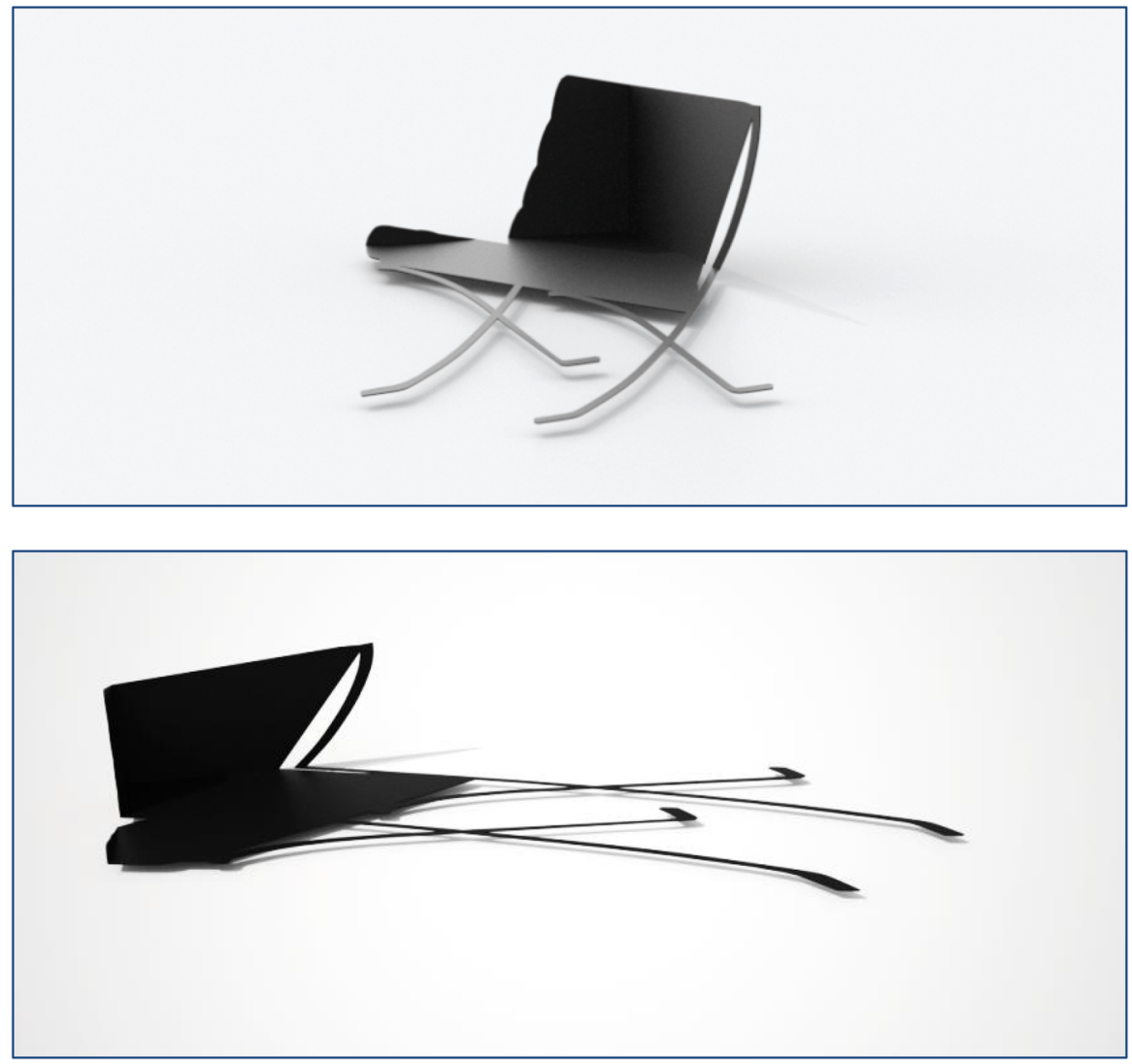

Fig. 12 Digital 3D model of an anamorphic shadow of Barcelona Chair modeled by the author. Image (a) seen from the vantage point, image (b) is seen from a different perspective

\section{Methodology and Digital Workflow}

Before setting up the teaching methodology for the aforementioned course, we studied some precedents in computational design and optical illusions, including some recent work presented in conferences such as Advances in Architectural Geometry, Sigraph and Nexus Network Journal. The course involved the analysis of famous case studies and the implementation of the knowledge gained in the projects undertaken during the semester. The project "Escher for Real" supervised by Professor Elber (Elber 2010) is a study on the "impossible drawings" of Escher, where tangible 3D models are created so that from a certain viewing direction they appear to be identical to the original 2D drawings, while from any other direction they are revealed to be valid yet deformed 3D geometric shapes. In their paper "Shadow Art" Mitra and Pauly utilize outline shadows as a source to try and synthesize 3D objects (Mitra and Pauly 2009). Since multiple shadow images often contradict each other, the authors have worked on a geometric optimization 


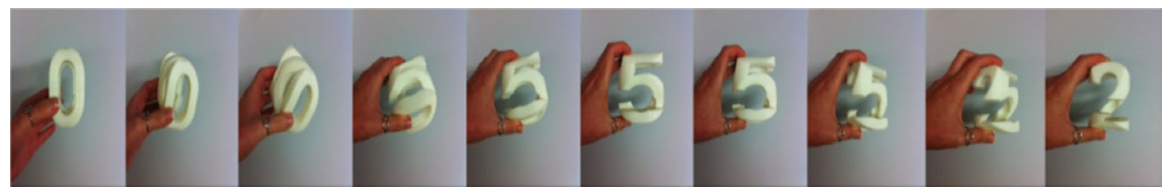

Fig. 13 3D printed Ambigram modeled and 3D printed by the author. The numbers 0,5 and 2 are visible if the Ambigram is seen from different vantage points

algorithm that computes a 3D shadow volume whose shadows best approximate the provided input images.

Unlike painting and other visual arts where non-realistic spaces can result from the interpretation and post-processing of images, our research agenda is focusing only on objects and spaces that are tangible, they can be defined and constructed geometrically with the use of CAD and even 3D printed (Fig. 13), yet they challenge our perception and understanding of space.

Departing from Hofstadter's iconic ambigrams, students studied possible ways to create solids that would generate a different shape according to the point of view. The method was based on the visual hull, introduced by Laurentini (1994) as the closest approximation of a 3D object that can be obtained from 2D silhouettes (the curves obtained from a parallel or perspective projection on a plane) with volume intersection approach. It is understood that an object cannot be reconstructed in precision based only on its $2 \mathrm{D}$ silhouettes. For the same reason a set of $32 \mathrm{D}$ silhouettes can correspond to several different 3D objects. Within this space of solutions, the students studied different extrusions and projections of a set of curves that were given as input. All students were assigned a different set of curves according to their matriculation number; the curves were generated from the outlines of the numbers. For the $3 \mathrm{D}$ experiments, the curves were constructed with a minimum number of control points, so that the resulting surfaces would be easy to manipulate through control point editing. The students implemented tools of geometrical Boolean operations (Union, Subtraction, Intersection), keeping or discarding parts of the solid that would correspond to the intended silhouette.

The process of geometrically creating an Ambigram usually followed certain steps, which involved operations in Rhinoceros 3D modeling software.

1. The planar curves generate 3 different solids through straight extrusion to define the potential space for the development of the Ambigram.

2. The solids are combined (through solid editing in Rhino) in one closed polysurface that can be viewed from different angles recreating the silhouette of the initial number-curves

3. Further operations of solid editing and reduction of material.

This second step might include several related processes; the geometrical problem is not to merely find the Boolean intersection of two or more solids. The understanding of topological relationships is of crucial importance, as the combination of topologically different solids would result in a hard-to-solve problem. However this task can be achieved through several different approaches, 
concerning the combination of shapes, hence the students would need to adopt solutions that would optimize the representational criteria. An added parameter to the Ambigram exercise was the intention to utilize as little 'material' as possible, thus reducing the volume where necessary without compromising the resulting silhouette. In that sense, the students would first obtain the visual hull and subsequently perform additional operations, to subtract volumes. "The visual hull is the maximal shape that projects consistently into a set of silhouettes, and is obtained by intersecting visual cones from the corresponding calibrated viewpoints" (Sinha and Pollefeys 2005, p. 2). Thus the perspective view of the Ambigram usually did not reveal the triple interpretation of the shape, but a rotation around it would make the whole process clear and visible to the spectator.

Based on the knowledge gained about projections, and inspired by the artwork of Thomas Quinn, Felice Varini, Marte Haverkamp and by the previously presented research undertaken by the author, the students carried out a second exercise which required that they digitally construct the shadow that an object casts on a given surface. The shadow was cast on multiple surfaces that would contain at least one change of plane.

The workflow here was similar to the ambigrams, with a clear sequence of geometric manipulations

1. Selection of the angle of the sun for the given model

2. Orientation of the camera parallel to the light beam

3. Creation of a Construction Plane in Rhino perpendicular to the light beam

4. Setting the camera as axonometric (parallel view in Rhino, CPlane top) as opposed to the default perspective, so that the projected image on the background surface coincides with the silhouette of the object

5. Extracting the silhouette of the object for the given camera view

6. Projecting the silhouette onto the "built environment" where the shadow would be cast according to the given Construction Plane.

As a last step before rendering the results, the students would give thickness to their models, through offsetting to solid or extrusion of a surface, thus, based on the shadow, they would create a complete new object that may or may not resemble the initial model.

Both processes described above relate to a design approach where 2D representation is transferred and transformed into 3D representation and back to 2D when projected on a surface. During these transformations, the 3D objects either retain or lose some of their characteristics, for example maintaining their silhouettes but losing their volumetric information, thus leading to completely new objects that trick our perception of space, resulting in different illusions according to the point of view, as the visually perceived images are different from the objective reality.

The third and last task of MySpace challenged students to use the knowledge gained to create their own optical illusion, mixing any of the methodologies seen above to create a $3 \mathrm{D}$ composition. The results obtained ranged from shadow experiments to sculptural representations (Figs. 14, 15, 16, 17, 18). Some of them were even animated to show the different points of vantage. Without any doubt, this sort of experiment wouldn't have been possible without the use of computational tools. 


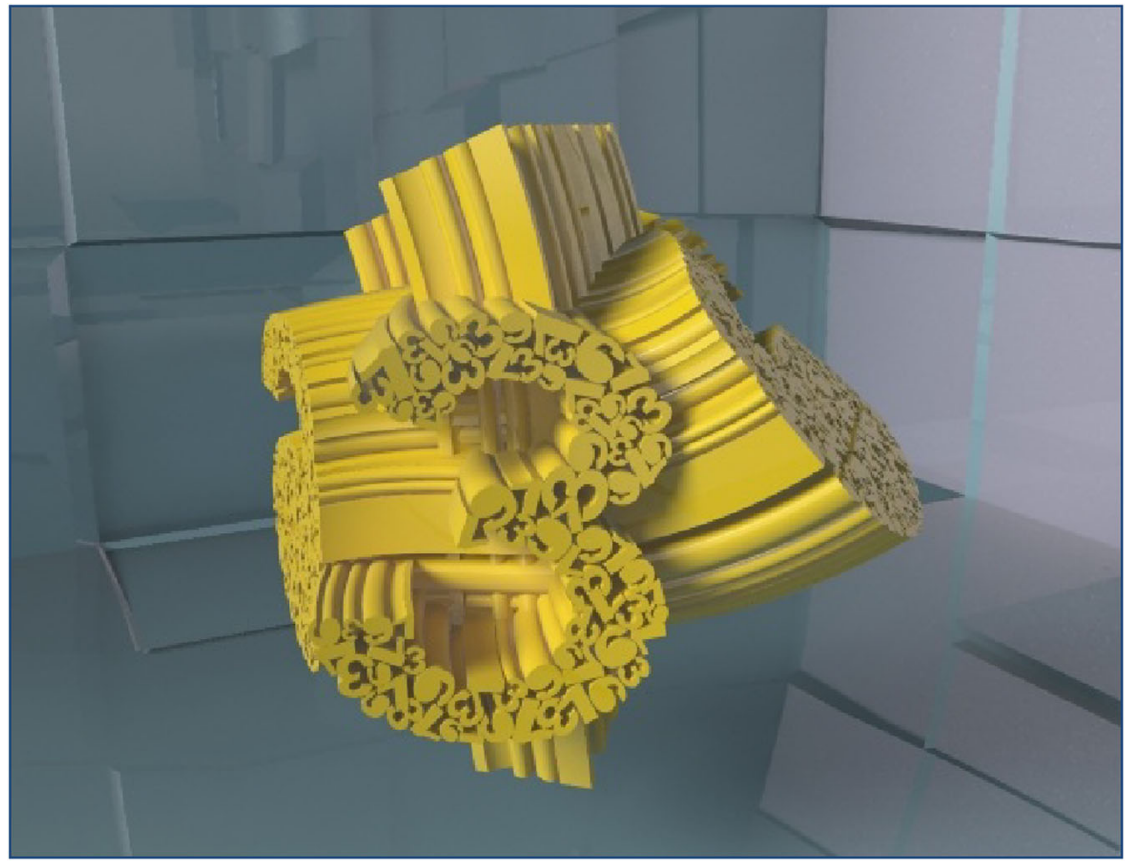

Fig. 14 Ambigram modeled by student Mak Pavelic at Graz University of Technology

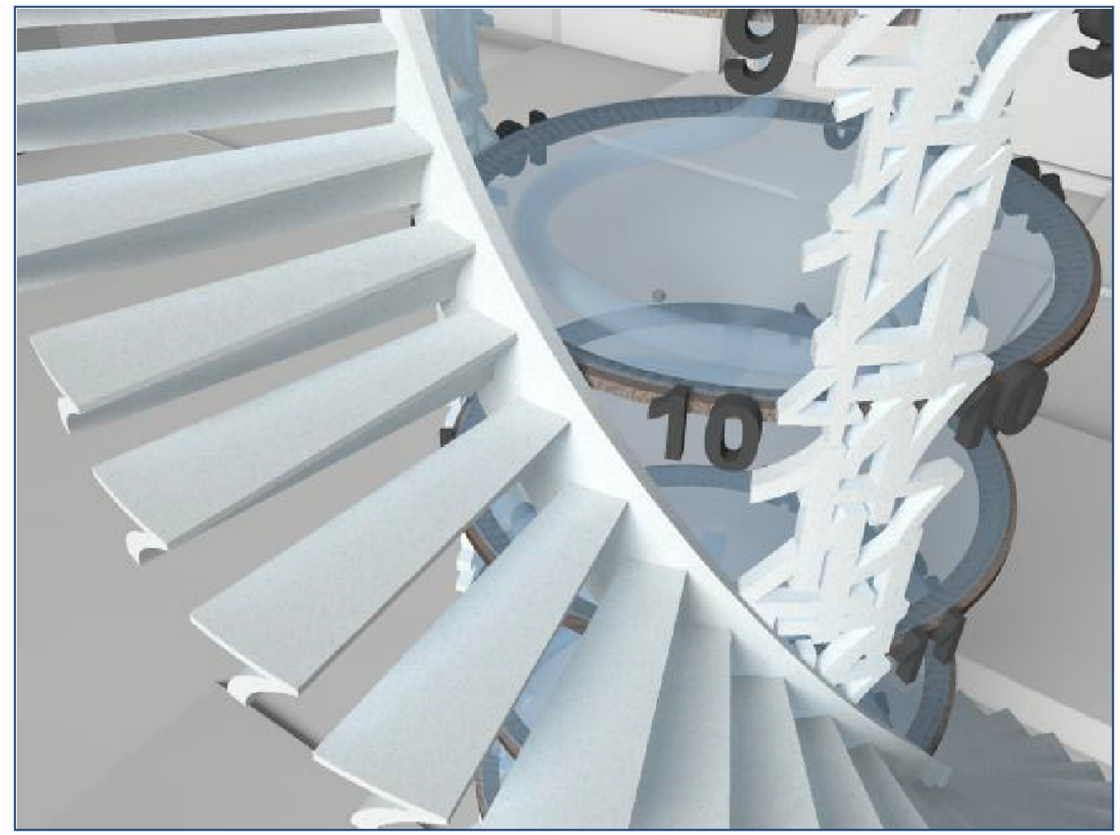

Fig. 15 Optical illusions modeled by student Paul Dominik Hoeber at Graz University of Technology 

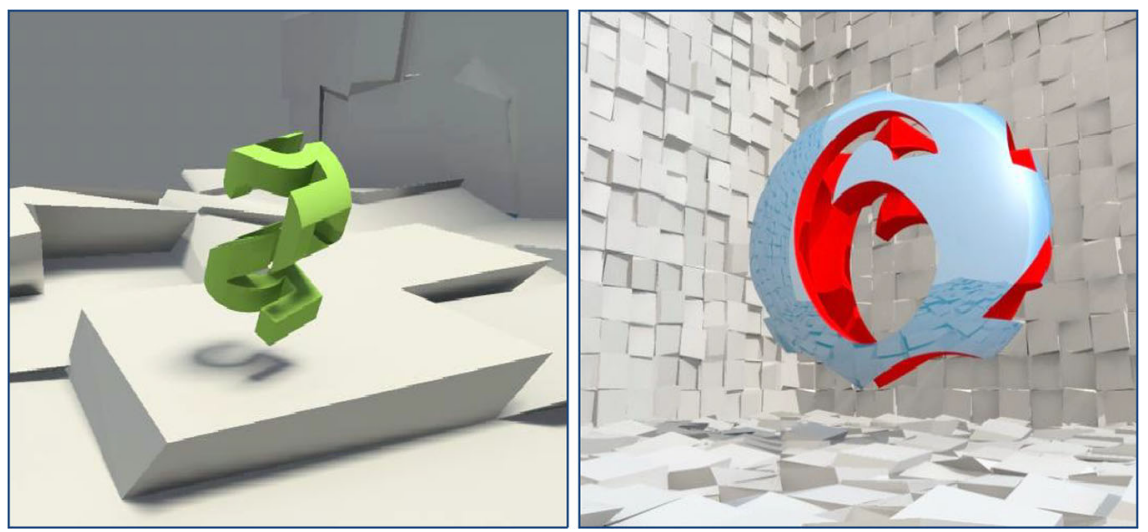

Fig. 16 Ambigrams modeled by students Alf Guggenberger and Caroline Brandstaetter at Graz University of Technology

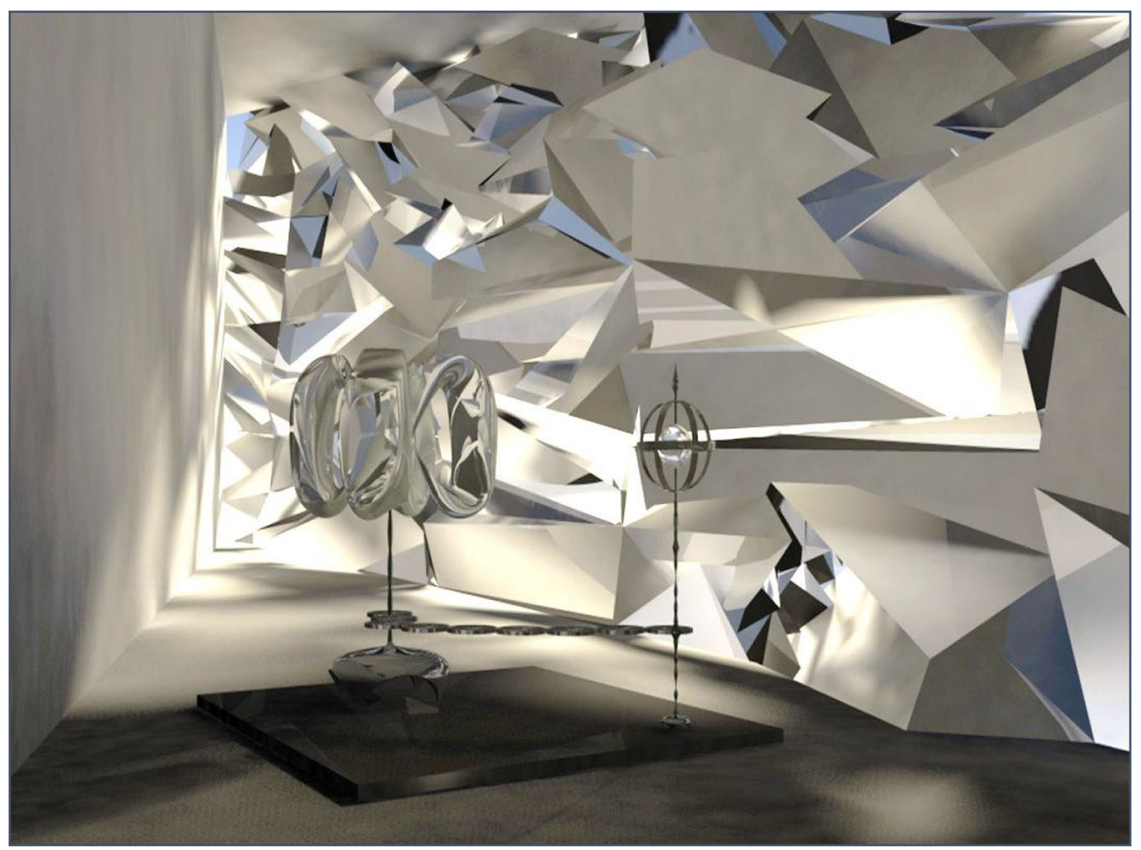

Fig. 17 Optical illusions modeled by student Daniel Plazza at Graz University of Technology

The use of digital media has liberated the creativity of the designers, as Elber remarks "The synergy between the sciences and the arts, and between geometric modeling and the plastic art in specific, is gaining a momentum in recent years" (Elber 2010, p. 176). Digital platforms such as Rhino, Maya, 3D max have become fundamental tools for art or architecture schools. Within the agenda of the course 


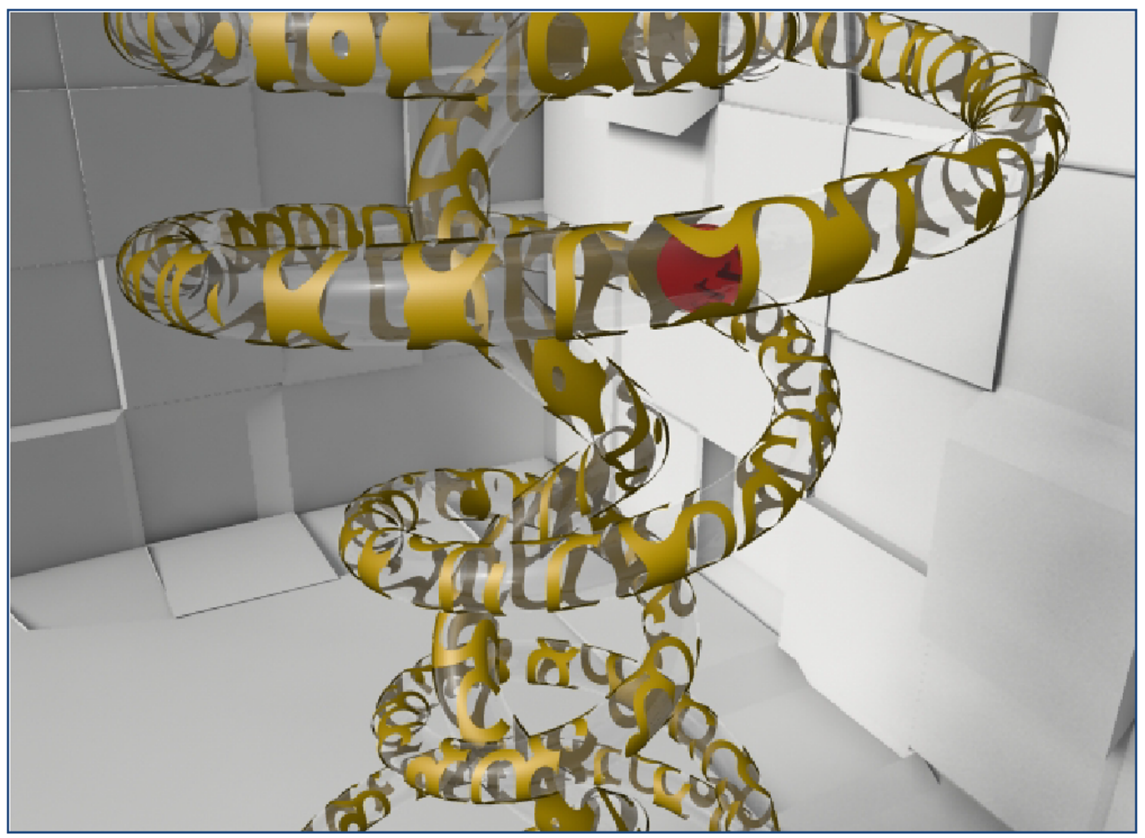

Fig. 18 Optical illusions modeled by student Teresa-Sixtina Maculan at Graz University of Technology

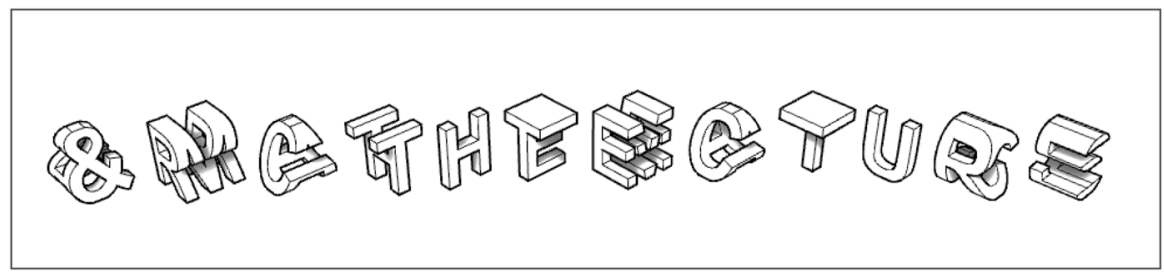

Fig. 19 Nexus Ambigram: The words architecture and mathematics can be read according to the point of view of the observer. The Nexus Ambigram was modeled by the author

'Digital Methods of Representation' students employed diverse computational tools for the creation and editing of their models. For the construction of Ambigrams, extrusions in different directions in combination with Boolean operations such as union, difference and intersection, were employed. To construct shadows they used projections, different viewport orientations, silhouettes and trims of the shaded polysurfaces. For the creation of anamorphic effects, the same geometric principles were in play, but very often the angle of projection was exaggerated in order to achieve the desired deformation of the idol. Through case studies and exercises the students mastered the necessary computational techniques and designed their own optical illusions challenging the limits of spatial perception and their understanding of computational geometry (Fig. 19). 


\section{Conclusion}

The use of geometrical curiosities to motivate learning has been also used in a simpler form for younger students, in secondary education. A recent project, the Geometry Playground, a traveling exhibition, initially presented at the Exploratorium Science Museum in San Francisco, encouraged visitors to use spatial reasoning, a kind of thinking where you make mental pictures of shapes and spaces. The students that visited the exhibition and actively explored different geometric experiences, such as anamorphic effects, moving geometries and tiling patterns, improved attitudes towards the focal topic (in this case geometry). According to the scientific reports of the Geometry Playground, studying the response of a number of students, the experience with hands-on geometry experiments helped "to bridge the gap between museum and school, perhaps ameliorating some negative associations students have with school geometry and potentially enhancing the educational effectiveness" (Dancu et al. 2009, p. 3). In the above report it is also discussed that student attitudes toward mathematics and geometry are "often negative, and those negative attitudes can have damaging effects on academic success and later career choices" (Dancu et al. 2009, p. 5). Geometry and computation is often a subject that receives negative preconception from students. There is a strong psychological component that highly influences the learning experience. Introducing a more playful yet challenging syllabus can aid students to discard this negative image and creatively engage in the mysteries of computational geometry. Evaluating the research and teaching experiences described in this paper, we can summarize and conclude with some basic remarks. During the course the students developed their abilities for spatial reasoning while improving their attitude towards geometry and mathematics in general. Such geometry experiments trigger students to think "outside the box" and instigate memorability; students acquire experience in problem solving and gain confidence with complex geometric issues. It was observed, and also verified through the evaluation of an exam on 3D modeling skills, that after the semester-long course, the students responded better to 3-dimensional problems and acquired a skillset that was utilized and further enriched during architectural studio courses.

From the obtained results, it was understood that the thematic area of shadows, anamorphic effects and optical illusions offered great opportunities for formal experimentation and geometry research. Students reacted very creatively to this rather abstract brief, very often exceeding the expectations and the academic requirements for this course. The participants of MySpace were equipped with a whole new skill-set about dealing with projections, different perspectives and shadow casting, while learning about precise 3D modeling and advanced surface editing. The design brief motivated students to think out of the box and create projects that are playful and challenging. The in-depth study of the work of the aforementioned artists opened the path for both students and educators to discover new methods and techniques; this experience highly influenced the way we perceive space. Just as the motto of the exhibition, 'nothing is as it seems', young architects should always question the relationship between the visually perceived image and 
the reality. Especially for contemporary architects, as the digital media very often generates ambiguities among design objects, it is important to be able to distinguish the difference between those designs that could be constructed and those that can only exist on the computer screen. Our encounter with digital objects will always generate certain ambiguities as well as learning opportunities. As developmental psychologist Edith Ackerman explains in her interview "Learning is all about moving in and out of focus, shifting perspective, and coming to see anew [...] it is like the art of living itself, as it is about navigating uncertainties rather than controlling what we cannot predict" (Ackermann and Hirschberg 2013, p. 78). Based on the results of the course, but also on the evolution of the ideas during the semester, we can say that the learning experience described above allowed students to learn, engage their minds and enrich their spatial cognition. As Ackermann affirms, it is important to establish a balance among the experience that the learners project and the intelligence gained by interaction with innovative tools.

\section{References}

Ackermann, E. and U. Hirschberg, U. 2013. Embodied Cognition and Reflective Practices. GAM Architecture Magazine 10: Intuition and the Machine. Graz: Ambra Verlag.

Čučaković, A. and M. Paunović. 2015. Cylindrical Mirror Anamorphosis and Urban-Architectural Ambience. Nexus Network Journal 17: 605-622. doi:10.1007/s00004-015-0239-7

Dancu, T., J. Gutwill, and L. Sindorf. 2009. Geometry Playground Pathways Study. San Francisco: Exploratorium Science Museum.

Di Paola, F., P. Pedone, L. Inzerillo and C. Santagati. 2015. Anamorphic Projection: Analogical/Digital Algorithms. Nexus Network Journal 17: 253-285. doi:10.1007/s00004-014-0225-5

Elber, G. 2010. Ortho-Pictures: 3D Objects From Independent 2D Data Sets In: Proceedings of Advances in Architectural Geometry, 175-192. Vienna: Springer.

Haverkamp, M. 2012. Shadow Construction. In: the official website of Marte Haverkamp, http://www. studiomarte.nl (accessed 15 October 2015).

Hofstadter, D. 1987. Ambigrammi (in Italian). Firenze: Hopeful-monster Editor.

Hofstadter, D. 2000. Gödel, Escher, Bach: An Eternal Golden Braid. London: Penguin.

Hunt, J.L. 2006. J-F Niceron's La Perspective Curieuse Revisited. In: Bridges London: Mathematics, Music, Art, Architecture, Culture, eds. Reza Sarhangi and John Sharp, 459-464. London: Tarquin Publications.

ILLUSION: Nothing is as it seems. 2013. Official Website of the Exhibition. https://dublin. sciencegallery.com/illusion (accessed 15 October 2015).

Laurentini, A. 1994. The Visual Hull Concept for Silhouette Based Image Understanding. IEEE Transactions on Pattern Analysis and Machine Intelligence. 16, 2: 150-162.

Mitra, N. and M. Pauly. 2009. Shadow Art. ACM Transactions on Graphics. 28, 5: Article 156.

Niceron, J.F. 1638. La Perspective Curieuse. Paris: Chez Pierre Billaine.

Polster, B. 2000. Mathemagical Ambigrams. In: Proceedings of the Mathematics and Art Conference 2000, 21-32. Bond University.

Sela, G., and G. Elber. 2007. Generation of View Dependent Models Using Free Form Deformation. The Visual Computer 23, 3: 219-229.

Sinha, S. and M. Pollefeys. 2005 Multi-View Reconstruction using Photo-Consistency and Exact Silhouette Constraints: A Maximum-Flow Formulation. In: Proceedings of the Tenth IEEE International Conference on Computer Vision (ICCV'05), 349-356. Washington: IEEE Computer Society. 
Ioanna Symeonidou is an architect, researcher and educator specializing in Digital Media, and an official trainer of Rhinoceros 3D in Europe. She has studied at the Aristotle University of Thessaloniki from where she graduated with distinction and obtained a postgraduate degree from the Architectural Association in London. Ioanna Symeonidou is currently teaching advanced design studios and digital design seminars at the Technical University of Graz in Austria and the International Hellenic University in Greece. She has collaborated with award winning design practices, Anamorphosis (Athens) Schwarzenbacher (Graz), HENN (Berlin) and FCC. SA (Spain). She has lectured and published internationally and has led Digital Design Workshops and Seminars in Greece, Spain, Austria, Portugal, Czech Republic including Aristotle University, IHU, Patras University, TU Graz, Czech Technical University of Prague, and FAUP-Porto. For her design and research work she has won numerous awards and grants. 